\title{
Critical branching-annihilating random walk of two species
}

\author{
Géza Ódor \\ Research Institute for Technical Physics and Materials Science, \\ H-1525 Budapest, P.O.Box 49, Hungary
}

\begin{abstract}
The effect of blocking between different species occurring in one dimension is investigated here numerically in the case of particles following branching and annihilating random walk with two offsprings. It is shown that in two dimension simulations confirm the field theoretical results with logarithmic corrections. In one dimension however if particles exhibit hard core interaction I confirm the very recent predictions of Kwon et al. [PRL 85, 1682 (2000)] that there are two different universality classes depending on the spatial symmetry of the offspring production characterized by $\beta_{S}=0.5$ and $\beta_{A}=2$. Elaborate analysis of simulation data shows that the order parameter exponent $\beta$ does not depend on initial conditions or on diffusion rates of species but strong correction to scaling is observed. By systematic numerical simulations the critical point properties have been explored and initial condition dependence of the dynamical exponents $Z$ and $\alpha$ is shown. In the case of a random initial state the particle-density decay at the critical point follows the $t^{-1 / 4}$ law with logarithmic corrections.
\end{abstract}

\section{INTRODUCTION}

Branching and annihilating random walk (BARW) process one of the simplest prototypes of a particle system exhibiting nonequilibrium phase transitions. For small creation/annihilation ratio the system evolves to a completely empty state from which no return is possible. This so called "absorbing state" lacks any fluctuations. For higher creation/annihilation rates, a steady state with some finite concentration of particles emerges with a phase transition. This phase transition has been investigated first by numerical simulations [1] [3] and later with field theoretical methods 4 . In general, we define annihilation of $k$ particles $k A \rightarrow \emptyset$ with the rate $\lambda$ and generation of $m$ offsprings $A \rightarrow(m+1) A$ with rate $\sigma$.

In $D>2$ dimensions the phase transition of these systems can be described by mean-field theory but in low dimensions the effect of fluctuations introduces more complex behavior. The $k=1,2 ; m=1$ case is the so-called directed percolation process; the continuous phase transition of it defines a robust universality class. According to the hypothesis of Janssen and Grassberger [5] [6] all continuous phase transitions to a single absorbing state in homogeneous systems with short-ranged interactions belong to this class provided there is no additional symmetry and quenched randomness present. As it was shown by Cardy and Taüber 4 this is the generic class for all critical models with $k=1,2 ; m=$ odd and indeed a large number of model systems [7] have been found to belong to this class.

If $k=m=2$ the parity of the number of particles is conserved locally under the reactions and another universality class appears: the so-called parity-conserving (PC) or BARW2 class. Particles following BARW2 dynamics may also appear as kinks between ordered domains in systems exhibiting two absorbing states [1,8 11]. For recent reviews see 112,13]. For $k>2$ the field theory does not predict any new universality classes.

Our knowledge of multiparticle systems is quite incomplete yet. A very recent paper of Janssen [14] shows that this usually leads to asymmetries between pairs of species and unidirectionally coupled systems arise. These systems have been shown to describe certain surface roughening processes 15, 16], for example.

The bosonic field theoretical work of [4] predicts a new universality class for BARW2 process of symmetric $N$ species (N-BARW2), exhibiting parity conservation $(m=k=2)$ of particles. They found that offspring creations of the same type like $A \rightarrow A A A$ are not relevant processes because new particles are annihilated immediately. In the case of branching processes like $A \rightarrow A B B$ and $A \rightarrow B B A$, all $N>1$ component models have the same Feynman diagrams as that of the $N \rightarrow \infty$ model and the critical point for $D \leq 2$ happens to be at $\sigma=0$ zero branching rate with the following critical exponents:

$$
\nu_{\|}=2 / d, \quad \nu_{\perp}=1 / d, \quad Z=2, \quad \alpha=d / 2, \quad \beta=1 .
$$

The critical exponents are defined by the usual way. The singular behavior of the spatial and temporal correlation lengths are characterized by

$$
\begin{aligned}
& \xi \propto \sigma^{-\nu_{\perp}}, \\
& \tau \propto \sigma^{-\nu_{\|}} .
\end{aligned}
$$

The anisotropy or dynamical exponent is $Z=\nu_{\|} / \nu_{\perp}$, while the particle density near the critical point obeys the scaling law

$$
\rho(t, \sigma) \propto t^{-\alpha} \phi\left(t \sigma^{\nu \|}\right)
$$

such that in the $t \rightarrow \infty$ steady state limit the order parameter scales as $\rho \propto \sigma^{\beta}$; hence 


$$
\beta=\alpha \nu_{\|}=\alpha Z \nu_{\perp}
$$

scaling relation holds. At the critical dimension $\left(D_{c}=\right.$ 2), logarithmic corrections to density decay are expected. For $D>2$ the critical point is also at $\sigma=0$ but governed by mean-field exponents [ [

In this work I investigated, numerically, the critical behavior of the 2-BARW2 model in one and two dimensions with the motivation that the existing field theories of reaction-diffusion models deal with a coarse grained, bosonic description of the particle system and therefore particle exclusion has not been taken into account. In a recent paper [17] we have shown that in the case of one-dimensional (1D) annihilating random walk of two species, the blocking between different species can cause marginal perturbations. Therefore it is an open question whether this can also be observed if we add particle creation to this process.

In a more recent paper Kwon et al. [18] argue based on local mean-field approximation that in this case new universality classes emerge indeed. I confirm the predictions of 18] for the order parameter exponent by precise numerical simulations and show that this exponent does not depend on initial-conditions or diffusion ratios of species. Furthermore I investigate the very nontrivial time dependence of this model and show numerical evidence for the initial condition dependence of the dynamical exponents and logarithmic corrections.

\section{DEFINITION OF THE MODEL}

A random sequential processes have been implemented in $D=1$ and $D=2$ dimensional lattices possessing periodic boundary conditions with one time step described as follows:

(1) A particle is selected randomly

(2) A nearest neighbor site is selected randomly

(3) With probability $1-\sigma$ the particle is attempted to move to the new site if that is empty

(4) If the new site is filled with a same kind of particle, both of them are removed

(5) With probability $\sigma$ two off-springs of different types from the ancestor are placed at two free nearest neighbor sites selected randomly.

In this way I investigate the same model as [18]. The field theoretical model of [4] also deals with the same particle reactions albeit without exclusion.

\section{SIMULATIONS FROM RANDOM INITIAL STATE IN TWO DIMENSIONS}

The initial conditions for systems with linear sizes $L$ have been set up in such a way that $L^{2} / 2$ randomly selected A or B particles are placed at random sites.

\section{A. Zero branching rate case $(\sigma=0)$}

First the field theoretical expectations [19,4] for the density decay $(\rho \propto \ln (t) / t)$ were tested. To see the logarithmic corrections, I plotted $\rho(t) \times t$ versus $\ln (t)$. As Fig. 11 shows the curves corresponding to different sizes show linear behavior for short times. Later the effect of finite system sizes causes this behavior to break down. The crossover happens for larger and larger times as $L$ goes to $\infty$.

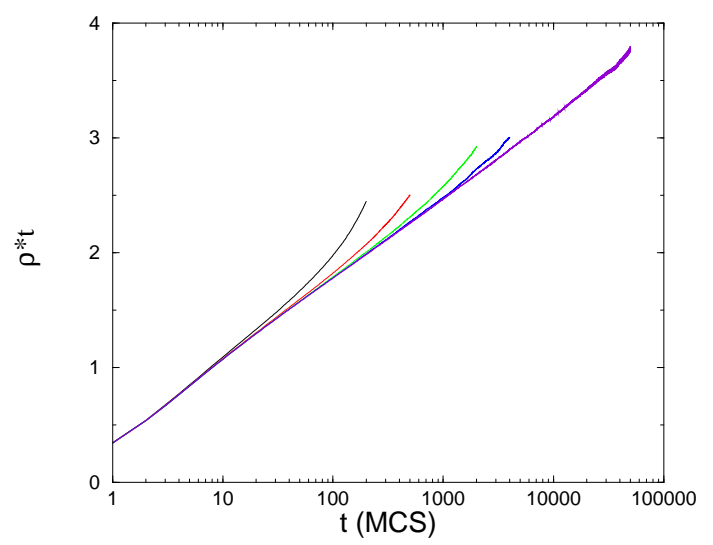

FIG. 1. Density decay (in $2 D$ ) of the total particle number for different system sizes: $L=16,32,64,128,512$ (from top to bottom). Throughout the paper $t$ is measured in units of Monte Carlo sweeps (MCS) of the lattices.

For the largest system investigated here $(L=512)$ a logarithmic fitting was applied in the $10<t<10^{4}$ region, resulting in

$$
\rho(t)=(0.442(2)+0.296(1) \ln (t)) / t
$$

Hence the field theoretical prediction $\alpha=1$ with logarithmic corrections has been confirmed by these simulations. The amplitude of the logarithmic term however differs from that of the pure annihilation model: $A_{2}=1 / 8 \pi$ [19]. One may speculate that in the two-component system this value is exactly $1 / \pi$.

\section{B. Off-critical case $(\sigma>0)$}

Traditional, direct simulations of off-critical exponents usually produce less precise estimates than cluster simulations for example, but now I could eliminate the two most important drawbacks of this method. The critical point is known to be exactly at $\sigma=0$; therefore this does not cause uncertainty in the estimation of the order parameter exponent. Furthermore I use precise analysis of the data by calculating local slopes that enables us to follow corrections to scaling.

The simulations with branching were performed in systems of linear sizes: $L=512$ in the neighborhood of the critical point $\left(\sigma_{c}=0\right)$ at $\sigma=0.0075,0.01 \ldots 0.17$ (Fig. 2). 
About 100 samples were run from independent random initial states and averaged over for each $\sigma$.

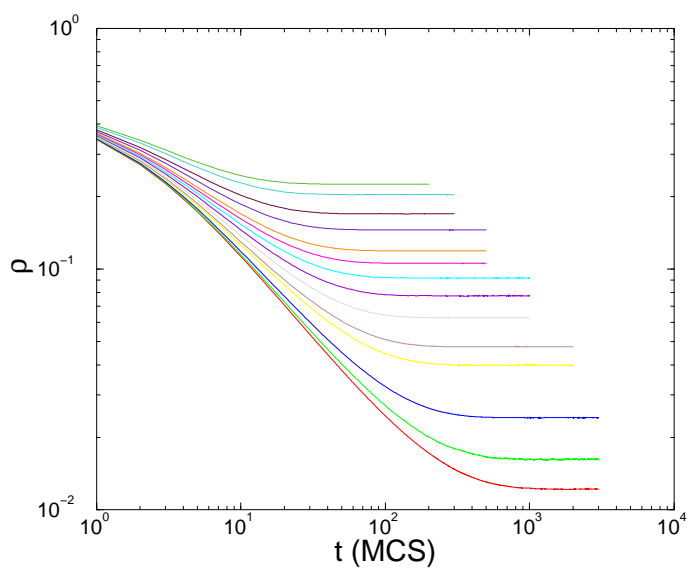

FIG. 2. Density decay (in $2 D$ ) of the total particle number for $\sigma=0.0075,0.01 \ldots 0.17$ (from bottom to top) in a system of size $L=512$.

From the results at the critical point (Fig. 1) one can see that this system size is large enough for finite size corrections to be negligible for $t<10^{4}$ MCS investigated here. That means we can assume that in my steady state simulations the correlation length does not exceed $L$. The particle density curves were averaged following saturation and the effective $\beta_{\text {eff }}(\sigma)$ exponents were calculated as

$$
\beta_{e f f}(\sigma)=\frac{\ln \rho_{i}-\ln \rho_{i-1}}{\ln \sigma_{i}-\ln \sigma_{i-1}},
$$

providing an estimate for the true asymptotic behavior of the order parameter

$$
\beta=\lim _{\sigma \rightarrow 0} \beta_{e f f}(\sigma) .
$$

By applying a linear extrapolation as in Fig. 3 a very good agreement with the field theoretical prediction for the order parameter exponent $\beta=1.003(3)$ can be found.

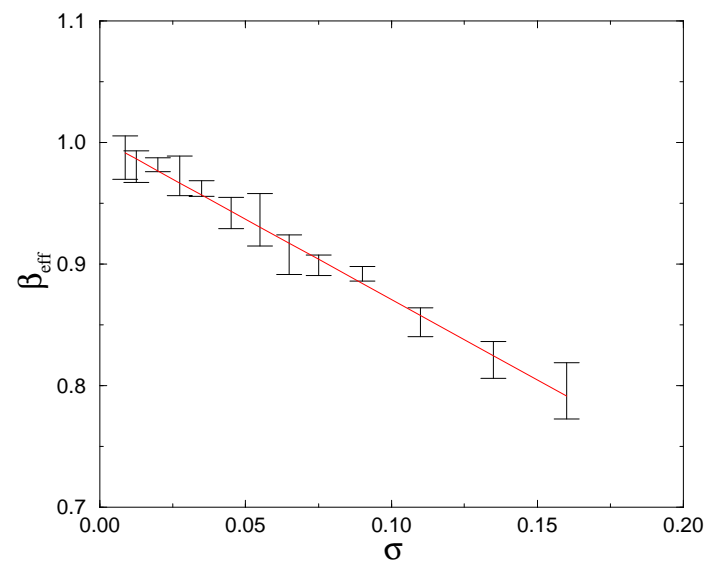

FIG. 3. Effective order parameter exponent $(\beta)$ results in $2 D$ systems of linear sizes $L=512$.

\section{SIMULATIONS IN ONE DIMENSION}

\section{A. The zero branching rate case $(\sigma=0)$}

In [17] we showed that in the case of special initial conditions

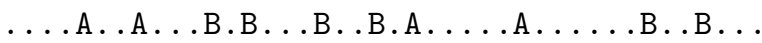

that assures that the time evolution never freezes (i.e. every particle has a neighboring particle of the same type to annihilate with) the density decays non-universally in an initial-condition dependent way. In this case particles can be regarded as boundaries of compact domains of different species that cannot overlap each other. This happens in the genaralized Domany-Kinzel cellular automaton, for example, 20].

The decay depends on the initial density (and hence on the initial interparticle separation length) of the species. We argued on the basis of symmetry considerations and by using an analogy to the works of Kaiser and Turban 21] that this decay follows the powerlaw with continuously changing exponents. We also showed that in the case of asymmetric initial conditions, where a small seed of B particles is inserted in the see of A's, the clustersurvival probability of B's (characterized by the exponent $\delta$ ) depends continuously on the initial density of A's similar to the case of the pair contact process [26]. Therefore in this work I investigated whether the static exponents show any initial-condition dependence as well and the form of the scaling law that connects them to the dynamic ones.

In the case of random initial conditions particles segregate into coarsening mosaic of alternating domains containing alternating sequences of A's and B's. By mapping this onto diffusion-controlled two-species annihilation $(A+B \rightarrow \emptyset)[22]$ Krapivsky and Redner argue that the particle density decays as $t^{-1 / 4}$ [23].

I tested this by simulating the process on $L=10^{5}$ lattices up to $t=4 \times 10^{6} \mathrm{MCS}$. The local-slopes curves of the density decay defined as

$$
\alpha_{e f f}(t)=\frac{-\ln [\rho(t) / \rho(t / m)]}{\ln (m)}
$$

(where usually I use $m=8$ ) converge to $\sim 0.25$ indeed (Fig. 4), but again like in 17] dependence on the initial conditions can be observed. Now however, the local slopes do not saturate in the $t \rightarrow \infty$ limit as in the case of "pairwise" initial conditions 17] but show logarithmically decreasing behavior as $t \rightarrow \infty$. This is not an artifact of the finite system sizes since the same behavior can be seen on much smaller $\left(L=10^{4}\right)$ lattices too. The origin of the logarithmic correction to scaling is not clear, but it is likely that marginal perturbation of species on each other causes it. Note that logarithmic correction to time dependence of the interparticle distances in case of 
the $A+B \rightarrow \emptyset$ model have also been shown by simulations [24] and explained to be the consequence of density inhomogeneity inside the domains.

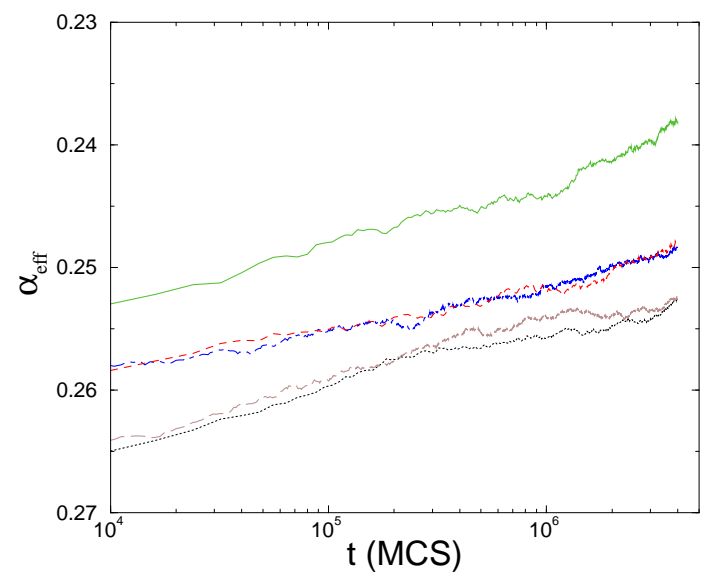

FIG. 4. Effective exponent of the particle decay in the one dimensional BARW2 with two species. The initial condition is randomly distributed A's and B's along the system of size $L=10^{5}$ sites. The solid line corresponds to initial densities: $\rho_{A}=\rho_{B}=0.375$, the dashed line to: $\rho_{A}=\rho_{B}=0.25$, the dotted line to $\rho_{A}=\rho_{B}=0.125$. The dot-dashed line corresponds to $L=10^{4}$ and $\rho_{A}=\rho_{B}=0.25$, while the long-dashed line corresponds to $L=10^{4}$ and $\rho_{A}=\rho_{B}=0.125$

This anomalous decay behavior cannot be described by the conventional field theoretical description of a particle system [4] in $D=1$ that omits site restrictions and predicts $\alpha=1 / 2$. Also the paper of Kwon et al. 18] based on local mean-field approximation does not show any initial-condition dependency but predicts simply the $\alpha=1 / 2$ result.

The dynamical exponent $Z$ has been determined through the measurement of the characteristic time $\tau(L)$ at the critical point. In this study I define $\tau(L)$ as the time it takes for some portion of the sample to enter the absorbing state. In the case of pairwise initial conditions this portion was $50 \%$ but in case of random initial conditions, where the evolution is very slow in finite systems, I chose this portion to be $10 \%$ in order to get results for $L>1000$ sizes. We expect the following finite size scaling at $\sigma=0$

$$
\tau(L) \propto L^{Z}
$$

The data points have been determined within the $L=$ $16-4096$ size region and to make precise extrapolation, local-slopes analysis has been performed. In Fig.5 I have plotted

$$
Z_{\text {eff }}(L)=\frac{\ln \tau_{i}-\ln \tau_{i-1}}{\ln L_{i}-\ln L_{i-1}},
$$

as a function of $1 / L$. As one can see, in the pairwise initial condition case $Z_{\text {eff }}$ converges to $Z=1.82(2)$ as $1 / L \rightarrow 0$ quite smoothly. However in the random initial condition case a huge correction to scaling can be observed and $Z_{\text {eff }}$ seems to extrapolate to $Z \sim 4.0(2)$, which is in accordance with the $t^{-1 / 4}$ density decay law.

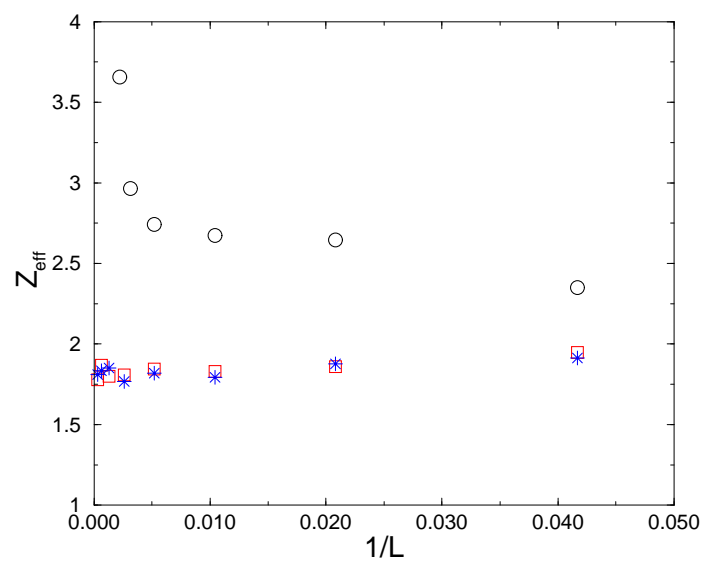

FIG. 5. $Z_{\text {eff }}$ vs $1 / L$ at $\sigma=0$. The square and star symbols correspond to pairwise initial conditions with $\rho_{A}=\rho_{B}=0.125,0.25$ respectively. The circles correspond to random initial conditions with $\rho_{A}=\rho_{B}=0.0625$.

The $Z=1.82(2)$ value is in agreement with our previous finding 17] where we showed that in the case of pairwise initial conditions the density decays as $\sim t^{-0.545(10)}$. We argued there that the interparticle distance of $\mathrm{AA}$ and $\mathrm{BB}$ pairs $l_{A A}=l_{B B} \propto \rho(t)^{-1}$ increases with the same power-law as the region of confinement that can be characterized with the exponent $1 / Z$. Now I could not obtain significant initial-condition dependence in $Z$ owing to the lack of such high precision simulations that we achieved in 17]. Note that the usual scaling relation between the single cluster spreading exponent $z$ (describing the mean distance from the origin $R(t) \propto t^{z / 2}$ ) and the dynamical exponent $Z=2 / z$ is violated. This may be understood if we realize that in the seed growing process there is no symmetric, mutual exclusion between different species therefore the exponent $z$ cannot describe the multisurface effect that arises in the case of pairwise initial conditions. Neither of these $Z$ values is in agreement with the assumptions of [18] and the field theoretical results for a 1D system without particle exclusion [4].

\section{B. The off-critical case $(\sigma>0)$ with symmetric (static) branching}

The effect of the spatial symmetry in simple onedimensional BARW processes was first investigated in [25] and it was shown that reflection-symmetric (static) branching causes reentrant phase diagrams unlike the asymmetric (dynamic) branching process. First with the same special, pairwise initial conditions as in our recent paper [17], I performed steady state simulations such that offsprings are generated symmetrically on the left and the right sides of parents $A \rightarrow B A B, B \rightarrow A B A$ (static branching). Kwon et al. [18] reported different critical 
behavior than what was expected by applying the results of the bosonic field theory [4] for this particle system.

Similar to the two-dimensional case, the density decay was followed carefully and averaging was done within a long time period, following the steady state having been built up. Figure 6 shows my simulation results of the order parameter $\beta$ in cases of different diffusion rates. As one can see, all the curves show strong (nonmonotonic) correction to scaling in the neighborhood of $\sigma=0$ but they all tend to the value $\beta=0.5$. The overshooting of the effective exponent signals possible logarithmic correction to scaling. The relaxation times to steady state were $t<10^{5}$ MCS in these simulations.

I found that in the neighborhood of the critical point the steady state concentration is unchanged if I use larger lattice sizes $\left(L=4 \times 10^{5}\right.$ instead of $\left.10^{5}\right)$, therefore the strange shape of the curves cannot be an artifact of the finite system size of the simulations. Note, that had we done a simple fitting onto the data points we could have estimated $\beta \sim 0.55$ instead of $\beta=0.5$.

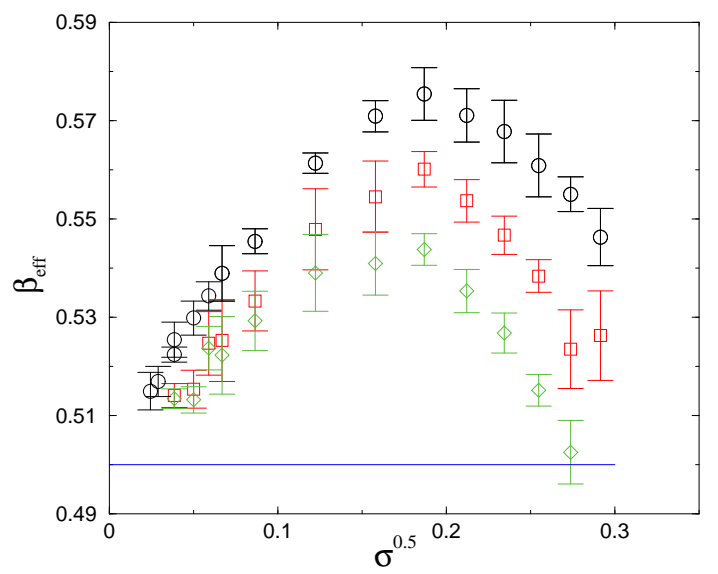

FIG. 6. Effective order parameter exponent $(\beta)$ results for the symmetric branching case. Circles correspond to the equal diffusion case, squares and triangles to $d_{A}=d_{B} / 2$ nonequal diffusion rate case of species.

The results are plotted as functions of $\sigma^{0.5}$ determined experimentally such that the curves approach $\sigma=0$ in an asymptotically straight line manner. This means that the leading correction to the scaling exponent is $\sim 0.5$. As one can see, the curves converge to $\beta=0.50(1)$ in agreement with the predictions of 18]. The simulations with other initial densities and random initial distributions of particles resulted in the same steady state values and hence $\beta$ is unchanged.

Comparing the $Z$ 's of the previous section with the $\nu_{\perp}=1 / 2$ of [18] determined by static finite size scaling for the symmetric branching model we predict for pairwise initial conditions $\nu_{\|}^{p}=Z \nu_{\perp}=0.915(2)$ and $\nu_{\|}^{r}=2.0(1)$ for random initial conditions.

The scaling law of density is fulfilled within error limits $\alpha^{p} \simeq 0.545 \simeq \beta / \nu_{\|}^{p}=0.5 / 0.915$ for the pairwise initial case, $\alpha^{r} \simeq 0.25 \simeq \beta / \nu_{\|}^{r}=0.5 / 2$ for random initial conditions.

The scaling form (4) can be checked by plotting $\rho t^{\alpha}$ against $t \sigma^{\nu \|}$. As Fig. 7 shows, one can obtain a fairly good data collapse with $\nu_{\|}^{r}=2$ and $\alpha^{r}=0.25$ (corresponding to random initial conditions) for large times. For small times the collapse is less good owing to the effect of logarithmic corrections to $\alpha^{r}$.

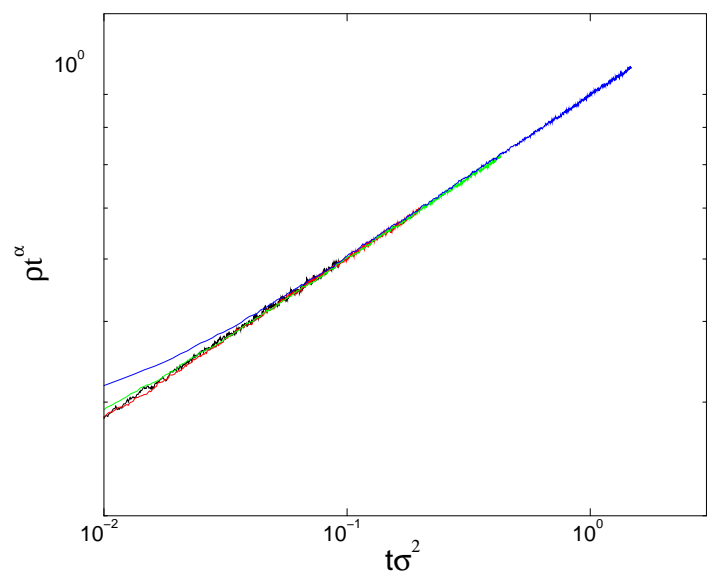

FIG. 7. Data collapse for off-critical simulations of the symmetric branching model according to the scaling form (4) with $\alpha^{r}=1 / 4$ and $\nu^{r} \|=2$. The different curves correspond to $\sigma=0.0005,0.001,0.002,0.005$ and random initial conditions.

For pairwise initial conditions a very good data collapse can be achieved with the standard scaling form (4) using $\alpha^{p}=0.545, \nu_{\|}^{p}=0.915$

\section{The off-critical case $(\sigma>0)$ with asymmetric (dynamical) branching}

For the same special, pairwise initial conditions as in our recent paper [17] I performed steady state simulations such that offsprings were generated on the left or right sides of parents $A \rightarrow A B B, A \rightarrow B B A, B \rightarrow B A A$ and $B \rightarrow A A B$. The relaxation time in this case was very long, typically $t>10^{6}$ MCS that prohibited getting closer to the critical point than $\sigma=0.03$. I performed these simulations in system sizes of $L=10^{5}$ sites and averaged over 100 samples.

As one can see in Fig. 8 the $\beta_{\text {eff }}$ extrapolates to 2.05(10) linearly in agreement with what was deduced from the local mean-field approximation by Kwon et al. [18. This value however, differs from the field theoretical predictions $(\beta=1)$ [ $\beta$ obtained for the coarse grained, bosonic description of the particle system, which neglects hard-core interactions. 


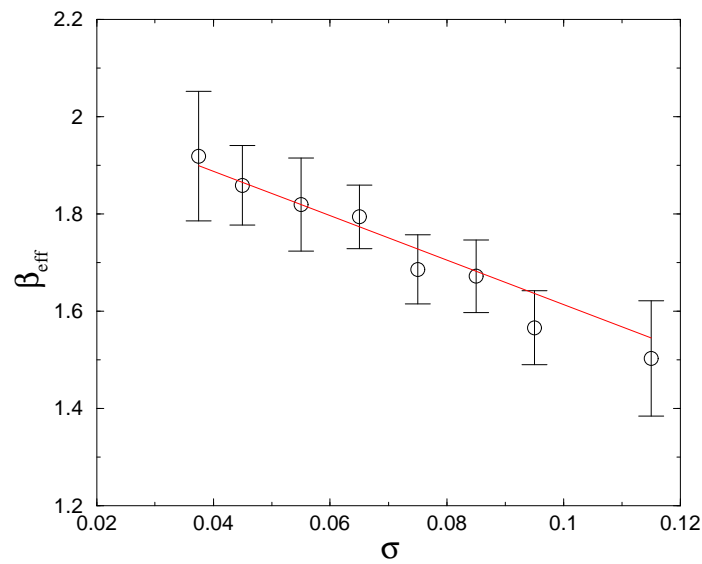

FIG. 8. Effective order parameter exponent $(\beta)$ results for the asymmetric branching case.

I investigated the decay by starting from other different initial conditions as well. The steady state concentration and hence the exponent $\beta$ was found to be the same, independent of the initial density of particles. In the case of random initial distribution of particles the relaxation was found to be even longer reflecting the fact that initial alternating sequences slow down the evolution to the steady state but the final concentration was the same. Again by comparing the $Z$ 's of Sec. IVA with $\nu_{\perp}=2$ of [18] determined by static finitesize scaling for the asymmetric branching process, we can predict for pairwise initial conditions $\nu_{\|}^{p}=Z \nu_{\perp}=3.66(2)$ and $\nu_{\|}^{r}=8.0(4)$ for random initial conditions. As in Sec. IVB the standard scaling relation of the density is fulfilled for both initial cases within error limits: $\alpha^{p} \simeq 0.545 \simeq \beta / \nu_{\|}^{p}=2 / 3.66$ $\alpha^{r} \simeq 0.25 \simeq \beta / \nu_{\|}^{r}=8 / 2$ and the scaling law can be confirmed by data collapse (see Fig. 9).

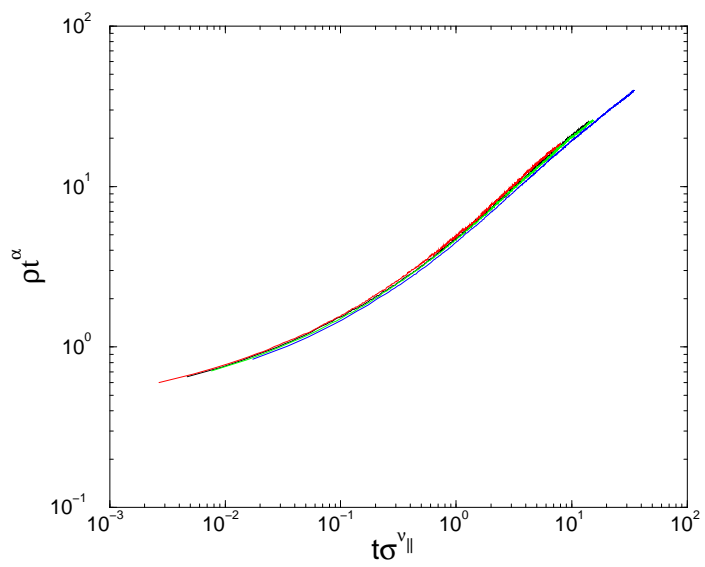

FIG. 9. Data collapse for off-critical simulations of the symmetric branching model according to the scaling form (何) with $\alpha^{p}=0.545$ and $\nu_{\|}^{p}=3.66$. The different curves correspond to $\sigma=0.03,0.035,0.04,0.05$ and pairwise initial conditions.

In the case of dynamic branching Kwon et al. 18 claim a slight dependence (a few percent) of the static exponents on the diffusion rates of different species. The relaxation time in this case is so huge that it hindered achieving such a precision by simulations.

\section{The off-critical case $(\sigma>0)$ without exclusion}

Finally I tested the field theoretical results in one dimension in such a way that I allowed particles of different types to exchange sites upon meeting. The steady state density has been determined for different values of $\sigma$ around zero in lattices with $L=2 \times 10^{4}$ size. As one can see in Fig.10 the middle curve (corresponding to this case) approaches to $\sigma=0$ linearly indeed in agreement with the $\beta=1$ field theoretical result. This can be verified by considering $\beta_{\text {eff }}$ as well.

\section{CONCLUSIONS}

In this study I confirmed numerically the field theoretical predictions for the two dimensional branching and annihilating random walk process of two species. The logarithmic corrections to the power-law density decay function have been determined.

In one dimension I gave numerical evidence that the bosonic renormalization group field theoretical predictions cannot be applied in contrast to widespread beliefs for systems with exclusion and the order parameter exponent depends on the spatial symmetry of the offspring production process. As Fig. 10 shows, the steady state density is much lower if offsprings are created on the same side of the parent against the case when they are separated by the parent resulting in different scaling exponents $\beta=2$ versus $\beta=1 / 2$.

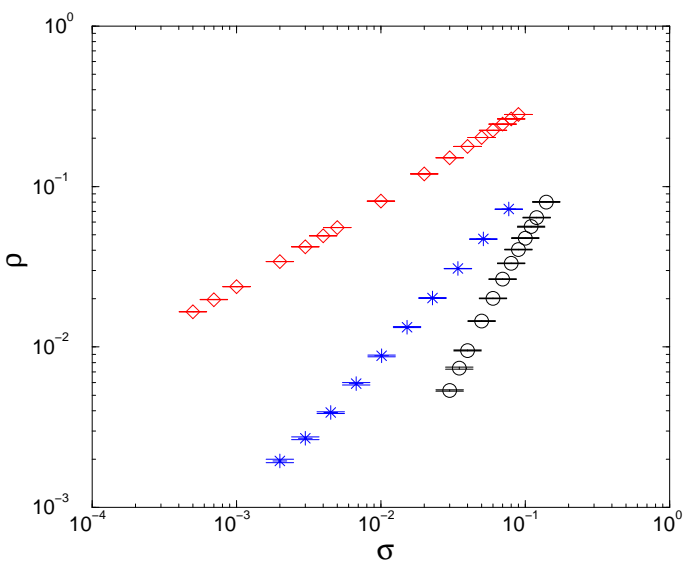

FIG. 10. Steady state density in the one-dimenional 2-BARW-2 model. Circles correspond to the asymmetric branching with $\beta=2$, diamonds to the symmetric branching with $\beta=1 / 2$ and stars to the model without exclusion $(\beta=1)$. 
This particle exclusion effect results in new universality classes in one dimension as summarized in Table I and the static exponents are insensitive to the initial conditions. In the case of static branching the precise numerical analysis of the order parameter data does not show dependence on the diffusion ratios either.

The density decay exponent starting from a random initial state $(\alpha)$ has been shown to agree with predictions of [23 but initial density-dependent logarithmic corrections to scaling can be observed. The dynamical exponent $Z$ has been found to depend on the initial conditions too. For pairwise initial conditions it is in agreement with our earlier study [17]. All these results for dynamical scaling deviate from those obtained by the continuum field theory and assumed in [18].

This study gives further evidence to the claims of Kwon et al. [18] that in one dimension a series of new universality classes emerge in nonequilibrium particle systems exhibiting hardcore interactions and continuous phase transitions. The relative simplicity of this model and the precision of the critical exponents obtained may provide a good starting point to more elaborate analytical investigations of systems with hardcore exclusion.

\section{Acknowledgments}

The author would like to thank Z. Rácz, S. Redner and U. Täuber for stimulating discussions. Support from Hungarian research fund OTKA (Nos. T-25286 and T23552) and from Bólyai (No. BO/00142/99) is acknowledged.

[1] P. Grassberger, F. Krause and T. von der Twer, J. Phys. A:Math.Gen., 17, L105 (1984).

[2] H. Takayasu and A. Yu. Tretyakov, Phys. Rev. Lett. 68, 3060, (1992).
[3] I. Jensen, Phys. Rev. E 50, 3623 (1994).

[4] J. L. Cardy and U. C. Täuber, J. Stat. Phys. 90, 1 (1998).

[5] H. K. Janssen, Z. Phys. B 42, 151 (1981).

[6] P. Grassberger, Z. Phys. B 47, 365 (1982).

[7] See examples in : J. Marro and R. Dickman, Nonequilibrium phase transitions in lattice models, Cambridge University Press, Cambridge, 1999.

[8] N. Menyhárd, J.Phys.A:Math.Gen., 27, 6139 (1994).

[9] M. H. Kim and H. Park, Phys. Rev. Lett. 73, 2579, (1994).

[10] H. Hinrichsen, Phys. Rev. E 55, 219 (1997).

[11] K. E. Bassler and D. A. Browne, Phys. Rev. Lett. 77, 4094 (1996).

[12] H. Hinrichsen, Adv. Phys. 49, 815 (2000).

[13] N. Menyhárd and G. Ódor, Brazilian J. of Physics 30, 113 (2000).

[14] H. K. Janssen, preprint, cond-mat/0006129.

[15] U.C. Täuber, M.J. Howard, and H. Hinrichsen, Phys. Rev. Lett. 80, 2165 (1998);

[16] H. Hinrichsen and G. Ódor, Phys. Rev. Lett. 82, 1205 (1999).

[17] G. Ódor and N. Menyhárd, Phys. Rev. E. 61, 6404 (2000).

[18] S. Kwon, J. Lee and H. Park, Phys. Rev. Lett. 85, 1682 (2000).

[19] B.P. Lee, J. Phys. A 27, 2633 (1994).

[20] H. Hinrichsen, Phys. Rev. E 55, 219 (1997).

[21] C. Kaiser and L. Turban, J. Phys. A:Math.Gen 27, L579 (1994); C. Kaiser and L. Turban, J. Phys. A:Math.Gen 28, 351 (1995).

[22] A. A. Ovchinnikov and Ya. B. Zel'dovich, Chem. Phys. 28, 215 (1978); S. F. Burlatskii and A. A. Ovchinnikov, Russ. J. Phys. Chem. 52, 1635 (1978); D. Toussaint and F. Wilczek, J. Chem. Phys. 78, 2642 (1983); K. Kang and S. Redner, Phys. Rev. A 32, 435 (1985); M. Bramson and J.L. Lebowitz, Phys. Rev. Lett. 61, 2397 (1988).

[23] P. L. Krapivsky and S. Redner, private communication.

[24] F. Leyvraz and S. Redner, Phys. Rev. A 46, 3132 (1992);

[25] S. Kwon and H. Park, Phys. Rev. E 52, 5955 (1995).

[26] I. Jensen and R. Dickman, Phys. Rev. E 48, 1710 (1993); I. Jensen, Phys. Rev. Lett. 70, 1465 (1993).

\begin{tabular}{|l|r|r|r|r|r|}
\hline \hline process & $\nu_{||}$ & $\nu_{\perp}$ & $Z$ & $\alpha$ \\
\hline nonblocking & 2 & 1 & 2 & $1 / 2$ & 1 \\
\hline symmetric & $2.0(1) / 0.915(2)$ & $0.50(1)$ & $4.0(2) / 1.82(2)^{*}$ & $0.25(1) / 0.55(1)^{*}$ & $0.50(1)$ \\
\hline asymmetric & $8.0(4) / 3.66(2)$ & $2.0(1)$ & $4.0(2) / 1.82(2)^{*}$ & $0.25(1) / 0.55(1)^{*}$ & $2.05(10)$ \\
\hline \hline
\end{tabular}

TABLE I. Summary of critical exponents in one dimension. The non-blocking data are quoted from [4]. The predictions for $\nu_{\perp}$ of the blocking models are cited from [18]. Data divided by "/" correspond to random vs. pairwise initial condition cases. Exponents denoted by ${ }^{*}$ exhibit slight initial density dependence [17]. 